\title{
Use of terrestrial gastropods (Cornu aspersum) as bioindica- tors of the environmental contamination status of the Sicilian Natural Parks to assess the contamination status of the pas- tures
}

Gianluigi Maria Lo Dico', Antonello Cicero², Giovanni Lo Cascio', Valentina Cumbo', Francesca Ornella Assiria', Andrea Macaluso', Gaetano Cammilleri', Rosa Filippi', Francesco Giuseppe Galluzzo', Barbara Randisi', Rosaria Collura', Vita Giaccone', Stefania Graci', Maria Drussilla Buscemi', Antonio Vella' \& Vincenzo Ferrantelli'

I Istituto Zooprofilattico Sperimentale della Sicilia “A. Mirri”, Via G. Marinuzzi 3, 90129 Palermo, Italy

${ }^{2}$ Dipartimento SASTAS, Università degli Studi di Messina, Polo dell’Annunziata, Messina, Italy

The use of land molluscs in biomonitoring can evidence interactions between the contaminant and the biotic sphere. The concentration of heavy metals were determined by ICP-MS. In areas where anthropic and industrial contamination persist, heavy metal levels were above the quantification limit; sampling in agricultural areas shows lower levels of heavy metals. The same trend of results can be found for the analysis of IPAs. Snails can be considered as bioindicators of pollutants living in close contact with the soil and ingesting plants with a high concentration of contaminants.

KEY WORDS

Snails; ICP-MS; heavy metals; IPA.

Received 22.11.2019; accepted 10.12.2019; published online 20.12.2019

Proceedings of the 4th International Congress on Biodiversity "Man, Natural Habitats and Euro-Mediterranean Biodiversity", November 17th-19th, 2017 - Malta

\section{INTRODUCTION}

Recently, with the transposition of Directive 2008/50 / EC (CAFE), attention has been paid to the use of bioindicators to assess changes in the quality of the environment. The organisms used as biomonitoring instruments should have specific sensitivity to certain environmental disturbances and are defined in the "biosensor" complex. Using bioindicator organisms it is possible to carry out an early environmental stress assessment and anticipate any interventions before major scale problems arise. As a result, they are an excellent monitoring tool. Some species of mollusks accumulate considerable amounts of metals and reflect bioavailable levels of the environment. For example, bivalve molluscs have long been used as bioindicators of contamination in aquatic environments rather than other animal groups. The use of land molluses in biomonitoring also allows for evidence of interactions between the contaminant and the biotic sphere, which can not be determined solely by soil chemistry and ambient air analysis, which only identifies the quantity of pollutant that may be present, providing accurate data on contamination. Spikes can come into contact with environmental pollutants following skin contact with the soil, ingestion of soil, vegetation, water, air inhalation. They are sufficiently fixed to provide information related to the contamination of a site; in case of 
bioaccumulation or physiological alterations, they would be used to evaluate in an integrated way the soil and air quality in terrestrial ecosystems. There are so many factors that affect the accumulation of metals, such as body weight, temperature, season and plants that feed. Many authors claim that molluscs of the same weight or size may be used for monitoring purposes and each metal has its own kinetics of intake and accumulation. Accumulation in the foot or the throat, the rate of growth and the mortality rate allow to define the environmental impact of contaminated soil on gastropods. To interpret the data of metal concentrations in gastropods, there is a classification of the concentrations of the metal found in the digestive gland based on the sampling environment. This classification, created by Pihan and also used by other authors, groups into concentrations in the digestive gland of the snails in the class and allows the site to be identified with greater contamination. This classification allows you to compare the results of your work with other existing ones in the literature and to understand the degree of contamination of the site being studied. In particular, the level of contamination of heavy metals $(\mathrm{Cd}, \mathrm{Pb}$, $\mathrm{Cu}, \mathrm{Zn}, \mathrm{Cr}$ ) and polycyclic aromatic hydrocarbons (IPA) will be assessed in two Sicilian Natural Parks and the Park of the Sicani Mountains of New Institution through the use of terrestrial gastropods as environmental bioindicators.

\section{MATERIAL AND METHODS}

The concentrations of heavy metals were determined by an ICP-MS (7700x series, Agilent Technologies, Santa Monica CA, USA) equipped with octopole reaction system (ORS3). The sample solutions were pumped by a peristaltic pump from tubes arranged on an autosampler ASX-500 Series (Agilent Technologies, Santa Monica (CA), USA) and then conducted on a quartz cyclonic spray chamber. A fast and efficient sample digestion was achieved by a microwave-assisted system Multiwave 3000 (Anton-Paar, Graz, Austria) equipped with a rotor for eight MF100 PTFE-TFM (poly-tetrafluoroethylene-tetrafluoroethylene) vessels. The determination of IPAs was conducted in GC-MS/MS (Trace 1310 coupled with TSQ Quantum XLS Thermo Fisher Scientific) with the PTV

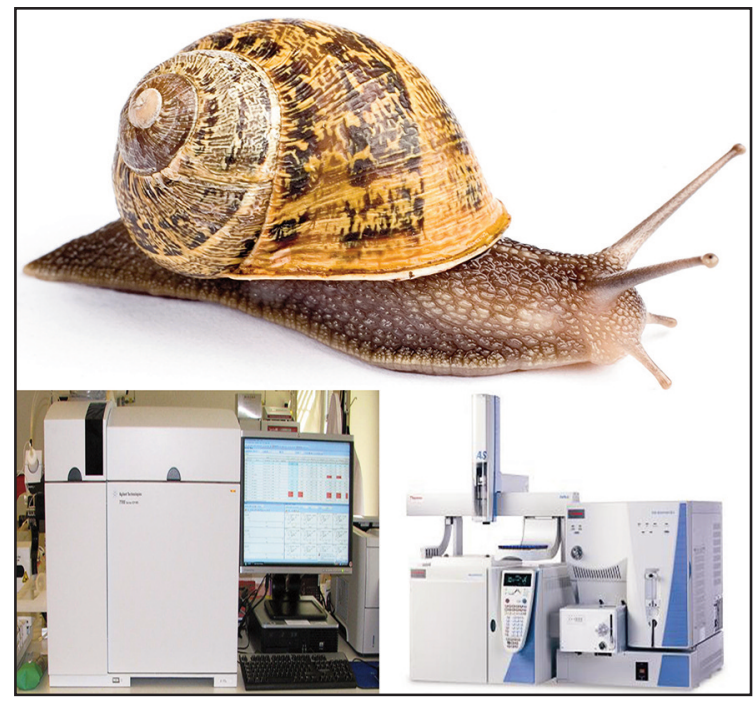

Figure 1. Cornu aspersum.

Figure 2, 3. ICP-MS Agilent series 7000 .

split/splitless injector. The GC-MS/MS analysis for the determination of PAH were carried out according to the protocol of Sapozhnikova et al. (2013).

The choice of using this analytical technique has been determined for the low detection limits that have been obtained.

\section{RESULTS AND DISCUSSION}

The data obtained from heavy metal analyzes show low levels of contamination in relation to the sampling area (see also Courdassier et al., 2002 and Amaral et al., 2004).

Areas where anthropic and industrial contamination persist, heavy metal levels above the quantification limit (LoQ) have been found; sampling in agricultural areas shows lower levels of heavy metals than LoQ. The same trend of results can be found for the analysis of IPAs. In fact, sampling in high-density areas and in industrial areas has yielded quantifiable results, unlike the results obtained by sampling in purely agricultural and mountainous areas.

\section{CONCLUSIONS}

The results obtained show that there is a transfer 
from the soil to the terrestrial gastropods of contaminants found. For this reason, snails can be used as bioindicators of the anthropic and industrial contamination levels of the territory where they were sampled. Furthermore, snails can be considered as carriers of pollutants living in close contact with the soil and ingesting plants with a high concentration of contaminants. Most contaminants are taken by gastroenteritis by ingestion. In particular, they focus on the digestive gland, which is the most important organ for both accumulation and detoxification. This organ is responsible for the production of digestive enzymes, absorption, digestion and endocytosis of food particles, as well as the storage and excretion of waste materials.

\section{REFERENCES}

Amaral A., Anselmo H., Toste Tristão da Cunha M. \& Rodrigues A., 2004. The connective tissue index of Helix aspersa as a metal biomarker. BioMetals, 17: 625-629.

Cœurdassier M., Gomot-de Vaufleury A. \& Lovy C., 2002. Is the cadmium uptake rom soil important in bioaccumulation and toxix effects for snails? Ecotoxicology and Environmental Safety, 53: 425-431.

Sapozhnikova Y. \& Lehotay S.J., 2013. Multi-class, multiresidue analysis of pesticides, polychlorinated biphenyls, polycyclic aromatic hydrocarbons, PBDEs and novel flame retardants in fish using fast, lowpressure gas chromatography-tandem mass spectrometry. Analytica Chimica Acta, 758: 80-92. https://doi.org/10.1016/j.aca.2012.10.034 
. 